\title{
Remaining challenges in cellular flavin cofactor homeostasis and flavoprotein biogenesis
}

\section{OPEN ACCESS}

Edited by:

Bruno Tota

University of Calabria, Italy

Reviewed by:

Tibor Kristian,

University of Maryland School of

Medicine, USA

Milagros Medina,

Universidad de Zaragoza, Spain

Sandro Ghisla,

University of Konstanz, Germany

*Correspondence:

Maria Barile,

Dipartimento di Bioscienze,

Biotecnologie e Biofarmaceutica, Università degli Studi di Bari Aldo Moro, via Orabona 4, I-70126, Bari,

Italy; Dipartimento di Scienze della

Vita, Istituto di Biomembrane e Bioenergetica, CNR, via Orabona, 4,

I-70126, Bari, Italy maria.barile@uniba.it

In Memoriam:

This paper is dedicated to the memory of Prof. Ernesto Quagliariello (1924-2004).

Specialty section:

This article was submitted to

Cellular Biochemistry,

a section of the journal

Frontiers in Chemistry

Received: 13 March 2015

Accepted: 05 April 2015

Published: 22 April 2015

Citation:

Giancaspero TA, Colella M, Brizio C

Difonzo G, Fiorino GM, Leone P, Brandsch $R$, Bonomi F, lametti $S$ and Barile M (2015) Remaining challenges in cellular flavin cofactor homeostasis and flavoprotein biogenesis.

Front. Chem. 3:30

doi: 10.3389/fchem.2015.00030
Teresa A. Giancaspero ${ }^{1}$, Matilde Colella ${ }^{1}$, Carmen Brizio ${ }^{1}$, Graziana Difonzo ${ }^{1}$, Giuseppina M. Fiorino ${ }^{1}$, Piero Leone ${ }^{1}$, Roderich Brandsch ${ }^{2}$, Francesco Bonomi ${ }^{3}$, Stefania lametti ${ }^{3}$ and Maria Barile ${ }^{1,4 *}$

${ }^{1}$ Dipartimento di Bioscienze, Biotecnologie e Biofarmaceutica, Università degli Studi di Bari Aldo Moro, Bari, Italy, ${ }^{2}$ Institut für Biochemie und Molekularbiologie, Universität Freiburg, Freiburg, Germany, ${ }^{3}$ Dipartimento di Scienze per gli Alimenti, la Nutrizione e l'Ambiente, Università degli Studi di Milano, Milano, Italy, ${ }^{4}$ Dipartimento di Scienze della Vita, Istituto di Biomembrane e Bioenergetica, CNR, Bari, Italy

The primary role of the water-soluble vitamin $B_{2}$ (riboflavin) in cell biology is connected with its conversion into FMN and FAD, the cofactors of a large number of dehydrogenases, oxidases and reductases involved in a broad spectrum of biological activities, among which energetic metabolism and chromatin remodeling. Subcellular localisation of FAD synthase (EC 2.7.7.2, FADS), the second enzyme in the FAD forming pathway, is addressed here in HepG2 cells by confocal microscopy, in the frame of its relationships with kinetics of FAD synthesis and delivery to client apo-flavoproteins. FAD synthesis catalyzed by recombinant isoform 2 of FADS occurs via an ordered bi-bi mechanism in which ATP binds prior to FMN, and pyrophosphate is released before FAD. Spectrophotometric continuous assays of the reconstitution rate of apo-D-aminoacid oxidase with its cofactor, allowed us to propose that besides its FAD synthesizing activity, hFADS is able to operate as a FAD "chaperone." The physical interaction between FAD forming enzyme and its clients was further confirmed by dot blot and immunoprecipitation experiments carried out testing as a client either a nuclear lysine-specific demethylase 1 (LSD1) or a mitochondrial dimethylglycine dehydrogenase $\left(\mathrm{Me}_{2} \mathrm{GlyDH}, \mathrm{EC}\right.$ 1.5.8.4). Both enzymes carry out similar reactions of oxidative demethylation, in which tetrahydrofolate is converted into 5,10-methylene-tetrahydrofolate. A direct transfer of the cofactor from hFADS2 to apo-dimethyl glycine dehydrogenase was also demonstrated. Thus, FAD synthesis and delivery to these enzymes are crucial processes for bioenergetics and nutri-epigenetics of liver cells.

Keywords: FAD, FAD synthase, flavinylation, lysine specific demethylase 1, dimethylglycine dehydrogenase, rat, nucleus, mitochondria, nutri-epigenetics

\section{Introduction}

The crucial role of the water soluble vitamin $\mathrm{B}_{2}$ or riboflavin (Rf) in cell metabolism is linked to Rf conversion into the enzyme cofactors flavin mononucleotide (FMN) and flavin adenine dinucleotide (FAD). In all the prokaryotic and eukaryotic cells the flavin cofactors ensure the functionality of hundreds of different flavoenzymes having dehydrogenase, oxidase, monooxygenase or 
reductase activities, and playing crucial roles in bioenergetics, photochemistry, bioluminescence, redox homeostasis, chromatin remodeling, DNA repair, protein folding, apoptosis, along with other physiologically relevant processes (Joosten and van Berkel, 2007). Thus, it is not surprising that deficiency of FAD-dependent enzyme and/or impairment of flavin homeostasis in humans and model animals have been linked to several diseases, such as cancer, cardiovascular diseases, anemia, abnormal fetal development, neuromuscular and neurological disorders (for rev. see Barile et al., 2013 and Refs therein). Therefore, understanding the membrane trafficking, homeostatic control, compartmentalisation, and turnover of Rf-derived flavin coenzymes within the cell is crucial to clarify the mechanism underlying the generation and maintenance of a normal cellular flavoproteome and of cellular metabolism. Some remaining challenges in cellular homeostasis of flavin cofactors and mitochondrial flavoprotein biogenesis will be dealt on in this paper.

Mammals must obtain $\mathrm{Rf}$ from the diet and, to a lesser extent, from intestinal microflora, whereas bacteria, protists, fungi, plants, and some animals can synthesize Rf from GTP and ribulose 5-P (Bacher et al., 2000). Dietary riboflavin is taken up in the human gastrointestinal tract by recently identified transporters, namely riboflavin transporters 1 (hRFT1) and 2 (hRFT2), that allow for vitamin concentration in the plasma and in blood cells (Haack et al., 2012). A third member of the Rf transporter family, namely riboflavin transporter 3 (hRFT3), is highly expressed in the brain (Yao et al., 2010; Patel et al., 2012; Foley et al., 2014). Mutations in hRFT2 and hRFT3 have been identified as in several individuals with a rare neurological disorder named Brown-Vialetto-Van Laere syndrome (Haack et al., 2012; Nabokina et al., 2012; Srour et al., 2014).

Once internalized in the cells, Rf conversion to cofactors occurs in two obligatory and ubiquitous steps, as schematised in Figure 1. The first enzyme of this pathway is riboflavin kinase (RFK, ATP: riboflavin $5^{\prime}$ phosphotransferase, EC 2.7.1.26), which transfers to Rf a phosphoryl group from ATP and forms FMN); the second enzyme-namely FAD synthase or FMN adenylyl transferase (EC 2.7.7.2, FADS or FMN-AT), previously known as FAD synthetase-is the enzyme responsible for FMN adenylation to FAD.

Compartmentalisation of flavin cofactor pools, generating the organelle-specific flavoproteome remains a question far from being elucidated and is sometimes a matter of debate (Lin et al., 2009; Barile et al., 2013; Kim and Winge, 2013; Lienhart et al., 2013). The main problem impairing an exhaustive comprehension of this issue derives from the necessity to coordinate FAD formation activities with events of cofactor assembly to client apo-proteins, that in eukaryotes occur mainly in mitochondria, but also in other compartments (Tu et al., 2000; Thorpe

Abbreviations: Rf, riboflavin; RFK, riboflavin kinase; FADS, FAD synthase; hFADS, human FAD synthase; hFADS1, human FAD synthase isoform 1; hFADS2, human FAD synthase isoform 2; FLAD1, FAD synthase gene; D-AAO, Damino acid oxidase; LSD1, Lysine specific demethylase $1 ; \mathrm{Me}_{2} \mathrm{GlyDH}$, dimethylglycine dehydrogenase; mtFSF, mitochondrial flavinylation stimulating factor; $\mathrm{PPi}$, pyrophosphate; LDH, lactate dehydrogenase. et al., 2002; Joosten and van Berkel, 2007; Stojanovski et al., 2008).

Subcellular localisation of FAD forming enzymes in mammalian, yeast and plant cells (Barile et al., 1993, 2000; Pallotta et al., 1998; Giancaspero et al., 2009) has been recently addressed at the molecular level (Bafunno et al., 2004; Torchetti et al., 2010; Liuzzi et al., 2012). These studies demonstrated that mitochondria possess their own FADS isoforms. In humans two different isoforms of human FADS were characterized, that are the products of alternative splicing generated from the human FLAD1 gene (Brizio et al., 2006; Galluccio et al., 2007). Isoform 1 and 2 are located in mitochondria and cytosol, respectively (Torchetti et al., 2010).

Moreover, a novel FADS localisation was found to be the nucleus of mammalian cells (Giancaspero et al., 2013). Additional sub-cellular localisations for FADS cannot be ruled out, and they could contribute to the maintenance of distinct flavin cofactor pools in different cellular compartments. The existence of a FAD forming pathway in the nucleus appears in line with the proposal of a possible control of FAD availability on the activity of the nuclear enzyme lysine-specific demethylase-1 (LSD1), which carries out the demethylation of di- and mono-methyllysine 4 in histone $\mathrm{H} 3$, an important epigenetic modification (Luka et al., 2011, 2014; Hino et al., 2012).

A second series of questions about the cellular biochemistry of Rf derived cofactors concerns the mechanism of release of the newly-synthesized FAD to "client" flavoproteins (Torchetti et al., 2011; Miccolis et al., 2014). All the recombinant FADSs produced up to now exhibit the ability to bind FAD - the product of their activity-tightly but not covalently (1 mol FAD: 1 mol monomer), thus eliciting a typical flavoprotein absorbance spectrum, see Torchetti et al. (2011). Following SDS-PAGE the purified proteins still retain flavin fluorescence on the denaturing gel (Torchetti et al., 2011). FAD release from FADS is likely to be tightly controlled, and presumably requires welldefined conditions, including a correct redox state (Miccolis et al., 2014), the presence of an apo-protein accepting the cofactor and-possibly-some accessory proteins as reported for inorganic (Bonomi et al., 2008; Ye and Rouault, 2010) and organic cofactors (Padovani and Banerjee, 2009) in some human proteins. The hypothesis of a role of accessory/acceptor proteins in FAD release is consistent with (and-to some extent-supported by) the extremely low turnover number $\left(k_{c a t}\right)$ measured for the purified enzyme (Torchetti et al., 2011) as the isolated protein.

Experiments described here are aimed at (i) confirming the multiple sub-cellular localization of FADS in human cells; (ii) investigating in some detail the FADS kinetics; (iii) addressing the issue of FAD release to different "client" flavoprotein, differing in nature and strength of FAD linkage and sub-cellular localization. In each case we aim at demonstrating that a direct interaction between the FAD forming machinery and the client flavoprotein occurs, and that a direct cofactor transfer from the donor to the apo-protein acceptor occurs in a sort of "FADchaperoning" action played by FADS per se, without a third reactant. The involvement of additional factors in vivo-including Hsp60 and Hsp10-is also discussed. 


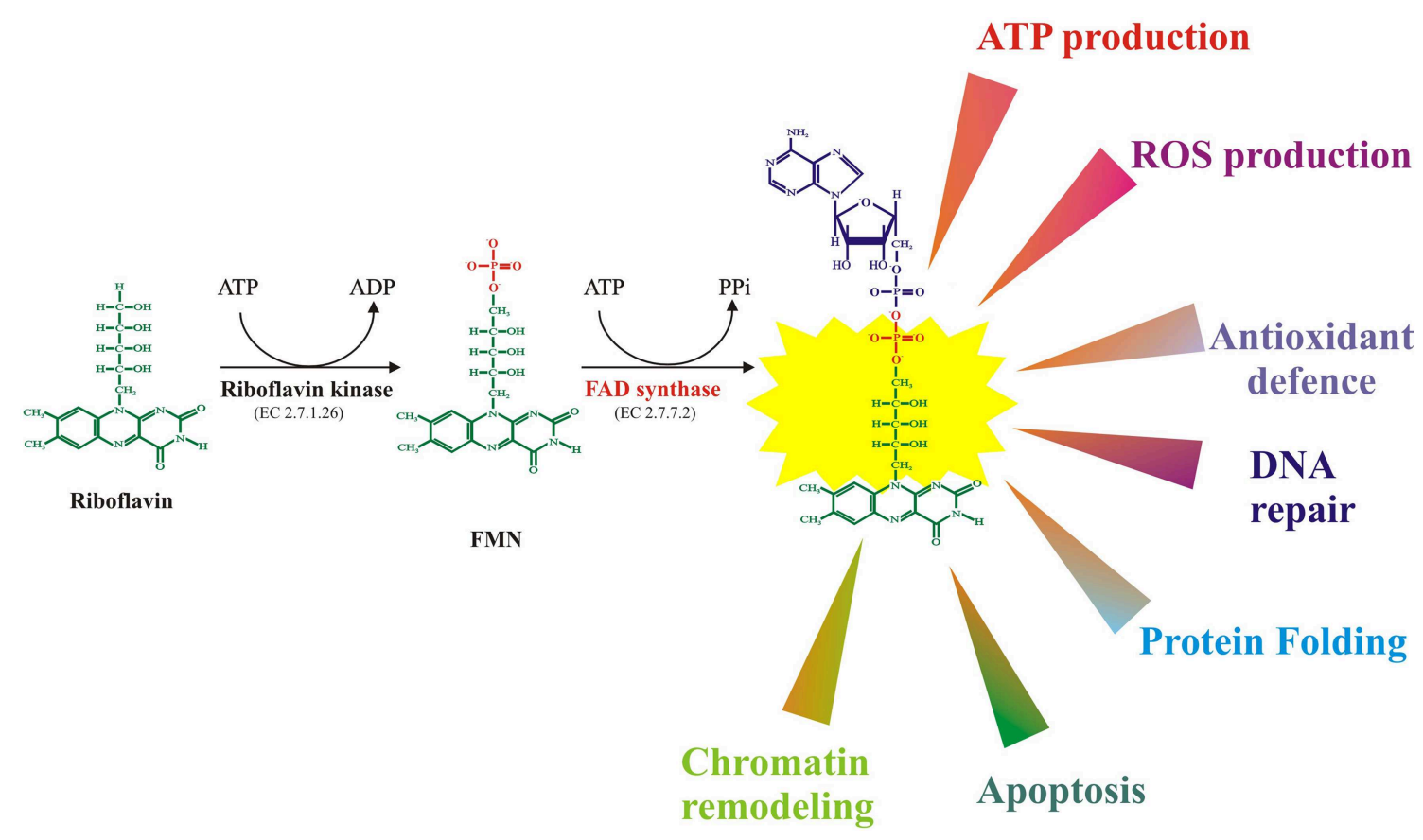

FIGURE 1 | FMN and FAD synthesis from riboflavin and main biological functions of flavoenzymes in mammalian cells.

\section{Materials and Methods}

\section{Materials}

All chemicals were of analytical or highest available grade and, unless otherwise stated, were obtained from SigmaAldrich. Polyvinylidene difluoride (PVDF) Hybond-P, Chelating Sepharose Fast Flow, and DEAE-Sephacel were from Amersham Ge-Healthcare. Reagents for protein assay were from Bio-Rad. Mouse anti-Hsp60 and Hsp-10 monoclonal antibodies were from Stressgen. Monoclonal mouse anti-LSD1 antibody was from Santa Cruz Biotechnology. Monoclonal mouse anti- $\beta$-actin antibody was from Abcam. Peroxidase conjugated anti-rabbit and anti-mouse IgG secondary antibodies were from Thermo Scientific. Alkaline phosphatase conjugated anti-rabbit and antimouse IgG secondary antibodies were from Sigma-Aldrich. Alexa Fluor conjugated anti-rabbit or anti-mouse IgG secondary antibodies were from Molecular Probes.

\section{Cell Immunofluorescence and Confocal Microscopy}

Immunofluorescence experiments were performed essentially as described elsewhere (Bruni et al., 2012; Cardone et al., 2012). Briefly, cells seeded on glass coverslips were fixed with $4 \%$ formaldehyde for $20 \mathrm{~min}$ and washed with PBS. After permeabilization (0.1\% Triton X-100 in PBS, $15 \mathrm{~min})$ and blocking $(0.1 \%$ gelatin in PBS, $1 \mathrm{~h}$ ), cells were incubated with the specific antiFADS rabbit antiserum (1:200 dilution). Nuclei were counterstained with Hoechst $2 \mu \mathrm{M}$ 33658. After washing, coverslips were mounted on microscope slides and confocal images were captured with a Leica TCS SP5 confocal microscope (Leica Microsystems, Mannheim) using a $63 \mathrm{X}$ (N.A. $=1.32$ ) oil immersion objective, a $100 \mathrm{~mW}$ Argon laser (488 nm line) and a $50 \mathrm{~mW}$ diode ( $405 \mathrm{~nm}$ ) as in (Gerbino et al., 2012). Confocal images were analyzed using the software Fiji (Schindelin et al., 2012) as in Tonazzi et al. (2013).

\section{Purification of Recombinant hFADS2 and Rat $\mathrm{Me}_{2}$ GlyDHs}

Purified recombinant 6 His-hFADS2 was prepared as described in Torchetti et al. (2011). Protein concentration and FAD/ protein monomer ratio (i.e., the flavinylation level) were estimated by absorbance spectra, as in Torchetti et al. (2011). Purified recombinant 6 His-m- $\mathrm{Me}_{2} \mathrm{GlyDH}$ and $6 \mathrm{His}-\mathrm{p}-\mathrm{Me}_{2} \mathrm{GlyDH}$ were prepared in both their apo- and holo-form essentially as described in Brizio et al. (2004) and Brizio et al. (2008), respectively. The flavinylation level of purified recombinant $\mathrm{Me}_{2} \mathrm{GlyDH}$ was estimated by measuring the UV fluorescence of the SDS-PAGE separated protein band, due to covalently bound FAD cofactor, essentially as described in Brizio et al. $(2004,2008)$.

\section{Kinetic Analysis of 6His-hFADS2}

FAD synthesis rate was measured at $37^{\circ} \mathrm{C}$ in $1 \mathrm{~mL}$ of a standard reaction medium consisting of $50 \mathrm{mM}$ Tris- $\mathrm{HCl}, 5 \mathrm{mM} \mathrm{MgCl}$, $\mathrm{pH} 7.5$ in the presence of FMN and ATP added at the appropriate concentrations. The reaction was started with the addition of 6 His-hFADS2. FAD synthesis rate was determined by taking advantage of the differential fluorimetric properties of FAD with respect to FMN (Barile et al., 1997). Fluorescence time courses (excitation at $450 \mathrm{~nm}$ and emission at $520 \mathrm{~nm}$ ) were followed in a LS50 Perkin-Elmer spectrofluorimeter. In each experiment, FAD and FMN fluorescence were calibrated individually using standard solutions whose concentration was calculated by using 
$\varepsilon_{450 \mathrm{~nm}}=12.2 \mathrm{mM}^{-1} \cdot \mathrm{cm}^{-1}$ for $\mathrm{FMN}$ and $11.3 \mathrm{mM}^{-1} \cdot \mathrm{cm}^{-1}$ for FAD. Under the experimental conditions used here, the FAD fluorescence constant $\left(K_{\mathrm{FAD}}\right)$ was about 10 times lower than that of FMN $\left(K_{\mathrm{FMN}}\right)$. Thus, the rate of FAD synthesis, expressed as nmol FAD min $^{-1}$ (mg protein) ${ }^{-1}$, was calculated from the rate of fluorescence decrease, measured as the tangent to the initial part of the experimental curve by applying the equation described in detail elsewhere (Torchetti et al., 2011).

\section{Reconstitution of holo-DAAO Activity}

The reconstituted holo-D-amino acid oxidase (D-AAO, EC 1.4.3.3) activity, derived from FAD binding to the apo-D-AAO, was followed spectrophotometrically as described in Barile et al. (2000), using $25 \mathrm{mM} \mathrm{D}$-alanine as substrate. NADH oxidation in the L-lactate dehydrogenase (LDH, EC 1.1.1.27)-coupled reaction was followed spectrophotometrically at $340 \mathrm{~nm}$. The reaction rate was calculated by measuring the slope of the tangent to the linear part of the experimental curve. This rate was proven to be proportional to FAD concentration. Calibration curves were obtained by using standard FAD solutions.

\section{Preparation of Pure Nuclei from Rat Liver}

Nuclei were isolated from the liver of male Wistar rats by differential centrifugation in sucrose gradient essentially as in Giancaspero et al. (2013). The isolated nuclei were finally resuspended in nuclear buffer (20 mM Tris- $\mathrm{HCl}, \mathrm{pH} 7.5,0.5 \mathrm{mM}$ PMSF). The purity and functionality of the nuclear fractions were checked as in Giancaspero et al. (2013) by following (i) the increase in the enzymatic activity of the nicotinamide mononucleotide adenylyltransferase, a central player NAD biosynthesis, mostly expressed in the nucleus as isoenzyme 1 (Orsomando et al., 2012), (ii) the decrease in the enzymatic activity of LDH a marker enzyme of the cytosolic compartment. As a control immunoblotting assays of lamin A/C (a nuclear marker) and of tubulin (a cytosolic marker) were also carried out, as in Giancaspero et al. (2013).

\section{Preparation of Mitochondrial Matrix from Rat Liver and its Fractionation}

The mitochondrial matrix was obtained from purified rat liver mitochondria, as previously described (Barile et al., 1997) and fractionated by ionic-exchange chromatography according to Brizio et al. (2000) and Bafunno et al. (2004). Briefly, the mitochondrial matrix $(17.5 \mathrm{mg} / \mathrm{mL})$ was applied onto a DEAESephacel column $(2 \mathrm{~cm} \times 0.7 \mathrm{~cm})$, equilibrated with $50 \mathrm{mM}$ Tris$\mathrm{HCl}, \mathrm{pH}$ 7.5. The column was washed with the starting buffer $(1 \mathrm{~mL})$, then eluted with a discontinuous gradient of $\mathrm{NaCl}(50-$ $250 \mathrm{mM}$, step $50 \mathrm{mM}$ ) in the same buffer. For each step of the gradient two fractions of $0.5 \mathrm{~mL}$ were collected. Each chromatographic fraction was analyzed by western blotting using a rabbit polyclonal antiserum directed against human FADS (antiFADS, International Application number PCT/IT2009/000062 filed February 23, 2009 by Barile, Torchetti, Indiveri, Galluccio), as described in Brizio et al. (2013). Flavinylation Stimulating Factor (mtFSF) activity was assayed in each fraction here by measuring the increase of the flavin fluorescence of $\mathrm{Me}_{2} \mathrm{GlyDH}$ protein band separated by SDS-PAGE.

\section{Immunoblotting}

SDS-PAGE separated proteins were electro-transferred onto a PVDF membrane using a Trans-Blot semidry electrophoretic transfer cell (Sigma-Aldrich). The immobilized proteins were incubated overnight with a 3000-fold dilution of anti-FADS antiserum, as in Giancaspero et al. (2013). Other antibodies were used to reveal and quantify protein markers, including a mouse monoclonal anti- $\beta$-actin antibody $(1: 10,000$ dilution), and mouse monoclonal antibodies: anti-LSD1 (1:1000 dilution); anti-Hsp60 (1:1000 dilution); anti-Hsp10 (1:1000 dilution). Bound antibodies were visualized with secondary anti-rabbit or anti-mouse IgG antibodies conjugated with peroxidase (1:3500 dilution) or with alkaline phosphatase (1:3500 dilution).

\section{Dot Blot Experiments}

To identify the interaction between nuclear proteins and hFADS2, purified rat liver nuclei $(50$ or $100 \mu \mathrm{g}$ ) or purified recombinant 6 His-hFADS2 $(5 \mu \mathrm{g})$ were dot-blotted onto a nitrocellulose membrane. Where indicated, rat liver homogenate (homo, 50 or $100 \mu \mathrm{g}$ ), nuclei (50 or $100 \mu \mathrm{g}$ ) or nuclear buffer (none) were added to the dotted membrane. After $30 \mathrm{~min}$ incubation at $37^{\circ} \mathrm{C}$ the membranes were washed, saturated in blocking solution containing BSA $3-5 \%$ and probed with antibodies directed against human FADS (anti-FADS), LSD1 (anti-LSD1) or actin (anti-ACT1). Following a washing step, the bound antibodies were visualized with secondary anti-rabbit or anti-mouse IgG antibodies conjugated with alkaline phosphatase (1:3500 dilution). To reveal the interaction between $\mathrm{Me}_{2} \mathrm{GlyDH}$ and hFADS2, purified recombinant $\mathrm{Me}_{2} \mathrm{GlyDH}(1 \mu \mathrm{g})$ was dotted onto a nitrocellulose membrane. Purified recombinant 6His-hFADS2 $(3 \mu \mathrm{g}$, in HEPES buffer $40 \mathrm{mM}$, $\mathrm{pH} 7.5,5 \mathrm{mM} \mathrm{MgCl}_{2}$ ) was added to the dotted membrane in the presence of $5 \mathrm{mM}$ ATP and, where indicated, of $20 \mu \mathrm{M} \mathrm{FMN}$. After $30 \mathrm{~min}$ incubation at $37^{\circ} \mathrm{C}$, the membranes were washed and probed with the anti-FADS antiserum as above.

\section{Immunoprecipitation Experiments}

Anti-FADS antiserum was added to a Dynabeads ${ }^{\circledR}$ Protein G immuno-precipitation kit according to manufacturer's procedure and used to immuno-precipitate purified rat liver nuclei $(50 \mu \mathrm{g})$. The nuclear immuno-precipitated proteins were analyzed by immuno-blotting with the anti-FADS antiserum. The same PVDF membrane was tested with the anti-LSD1 and antiACT1 antibodies after performing a stripping procedure. The bound antibodies were visualized with secondary anti-rabbit or anti-mouse IgG antibodies conjugated with peroxidase (1:3500 dilution).

\section{Other Assays}

Protein concentration was assayed according to Bradford (1976), using bovine serum albumin (BSA), as standard. Quantitative evaluation of UV fluorescent and immuno-reactive protein bands was carried out by densitometric analysis using the Image lab software (BIORAD). 


\section{Results and Discussion}

\section{Subcellular Localization of FADS in Human Liver Carcinoma (HepG2) Cells}

As stated in the Introduction, the first challenge of this paper is to provide further evidence of multi-compartmentalisation of FADS in human cells. The presence of FADS in both the mitochondrion and nucleus was demonstrated for the first time in freshly isolated rat liver fractions (Giancaspero et al., 2013). To confirm the multi-compartmentalisation of FADS in human cells, immunofluorescence experiments were carried out on a human cell line derived from liver carcinoma, i.e., HepG2 (Figure 2). After fixation, permeabilization and incubation with the anti-FADS antiserum the immuno-complexes were visualized with a secondary antibody conjugated with Alexa Fluor 488 (Figure 2A). After nuclei counterstaining with Hoechst 33568 (Figure 2B), the coverslips were analyzed by confocal fluorescence microscopy. As apparent in Figures 2A,C, besides the expected cytoplasmic localization, a clear FADSimmunoreactivity was visible in the nucleus of HepG2 cells.

\section{Steady State Kinetics of Recombinant FADS}

Figure 3 reports the results of steady state kinetic experiments carried out with the recombinant 6 His-hFADS2, i.e., the cytosolic form of the enzyme (Torchetti et al., 2010), and aimed at addressing the second challenge, i.e., the enzyme mechanism of the synthase.

The dependence of the rate of FAD formation on FMN concentration (from 0.1 to $2 \mu \mathrm{M}$ ) at fixed concentrations of ATP was investigated by means of a rapid spectrofluorimetric assay (Barile et al., 1997). Results are reported according to a LinaeaweverBurk plot (Figure 3A). The dependence of reaction rate on ATP (ranging from 0 to $100 \mu \mathrm{M}$ ) at fixed FMN concentrations was investigated in parallel experiments (Figure 3B).

When FMN is varied at different ATP levels (from 1 to $100 \mu \mathrm{M}$ ), the pattern intersects on the vertical axis (Figure 3A). Thus, the $K m_{F M N}$ value decreases (from $8 \mu \mathrm{M}$ to $0.4 \mu \mathrm{M}$ ) at increasing ATP concentration. Conversely, when ATP is varied, the patterns intersects in the forth quadrant (Figure 3B). Thus,
$K m_{A T P}$ does not change significantly (at $\approx 15 \mu \mathrm{M}$ ) when increasing FMN concentration. A replot of the slopes of the LineaweverBurk plot (with FMN as variable substrate) vs. 1/[ATP] has a finite vertical intercept (inset of Figure 3A). A replot of the slopes of the Lineawever-Burk plot (with ATP as variable substrate) vs. $1 /[\mathrm{FMN}]$ goes through the origin, instead of having a finite vertical intercept. This patterns are consistent with a "sequential ordered bi-bi" mechanism for 6-His-hFADS2, and allow to define the order of substrate binding to the enzyme as ATP binding prior to FMN.

To define the order of product release the inhibition patterns of $\mathrm{PPi}$, one of the products in the FADS reaction, were studied according to a Dixon analysis (using fixed FMN concentration at $2 \mu \mathrm{M}$ in Figure 3C and using fixed ATP concentration at $10 \mu \mathrm{M}$ in Figure 3D). In Figure 3C a non-competitive inhibition pattern by PPi was observed when ATP is the variable substrate, since the patterns intersect on the horizontal axis. In Figure 3D the inhibition by PPi against FMN is consistent with competitive inhibition, since the pattern intersects in the forth quadrant. This mechanism is a special case of an ordered mechanism, named as the Theorell-Chance (see The enzyme- Kinetics and mechanism, Clealand, 1970). This allows to establish that PPi is the first product released immediately after FMN binds to the enzyme.

The results in Figure 3 clearly indicate that FAD synthesis occurs via an ordered bi-bi mechanism in which ATP binds prior to FMN, and pyrophosphate is released before FAD. A similar mechanism has been reported for other FADS, such as those from rat (Oka and McCormick, 1987) and C. glabrata (Huerta et al., 2009).

The slow release of FAD after PPi is consistent with (and to some extent supported by) the extremely low turnover number $\left(k_{c a t}\right)$ measured for the recombinant purified enzyme when monitoring FAD synthesis by direct and indirect assays and the observation that FAD remains tightly bound to the recombinant purified enzyme (Torchetti et al., 2011). Thus, our data indicate that FAD release may represent the rate-limiting step of the whole catalytic cycle and that the processes leading to FAD synthesis and delivery to client apoproteins may be tightly controlled by factors others than FADS itself.
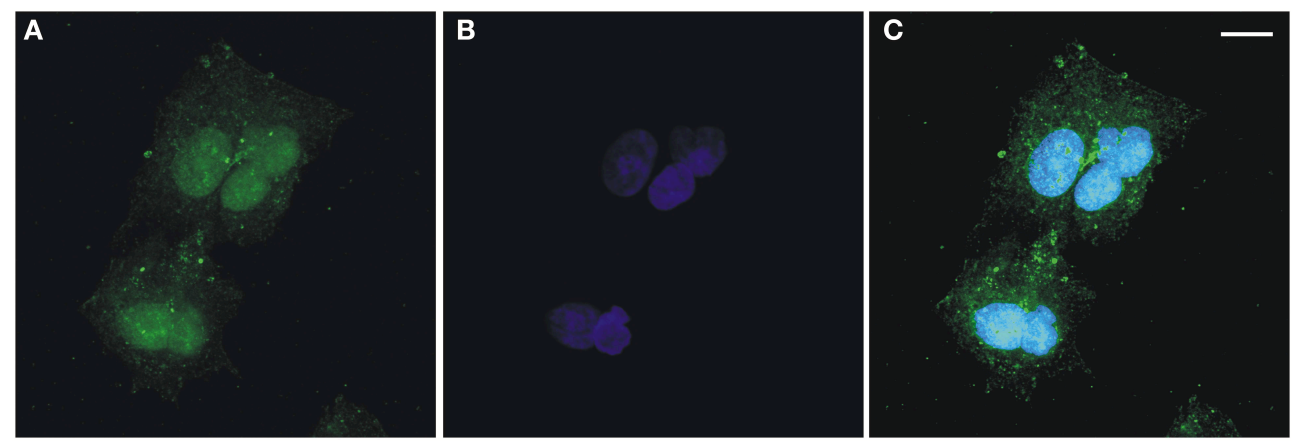

FIGURE 2 | Confocal microscopic analysis of hFADS subcellular localization in human liver carcinoma (HepG2) cells.

Maximum-intensity projections of confocal laser scanning image stacks of HepG2 cells labeled with the polyclonal anti-FADS antiserum followed by incubation with an Alexa Fluor 488-conjugated anti-rabbit antibody (green, A). Nuclei were stained with Hoechst 33658 (blue, B). The overlay of the two images is represented in (C). Scale bar = $10 \mu \mathrm{m}$. 

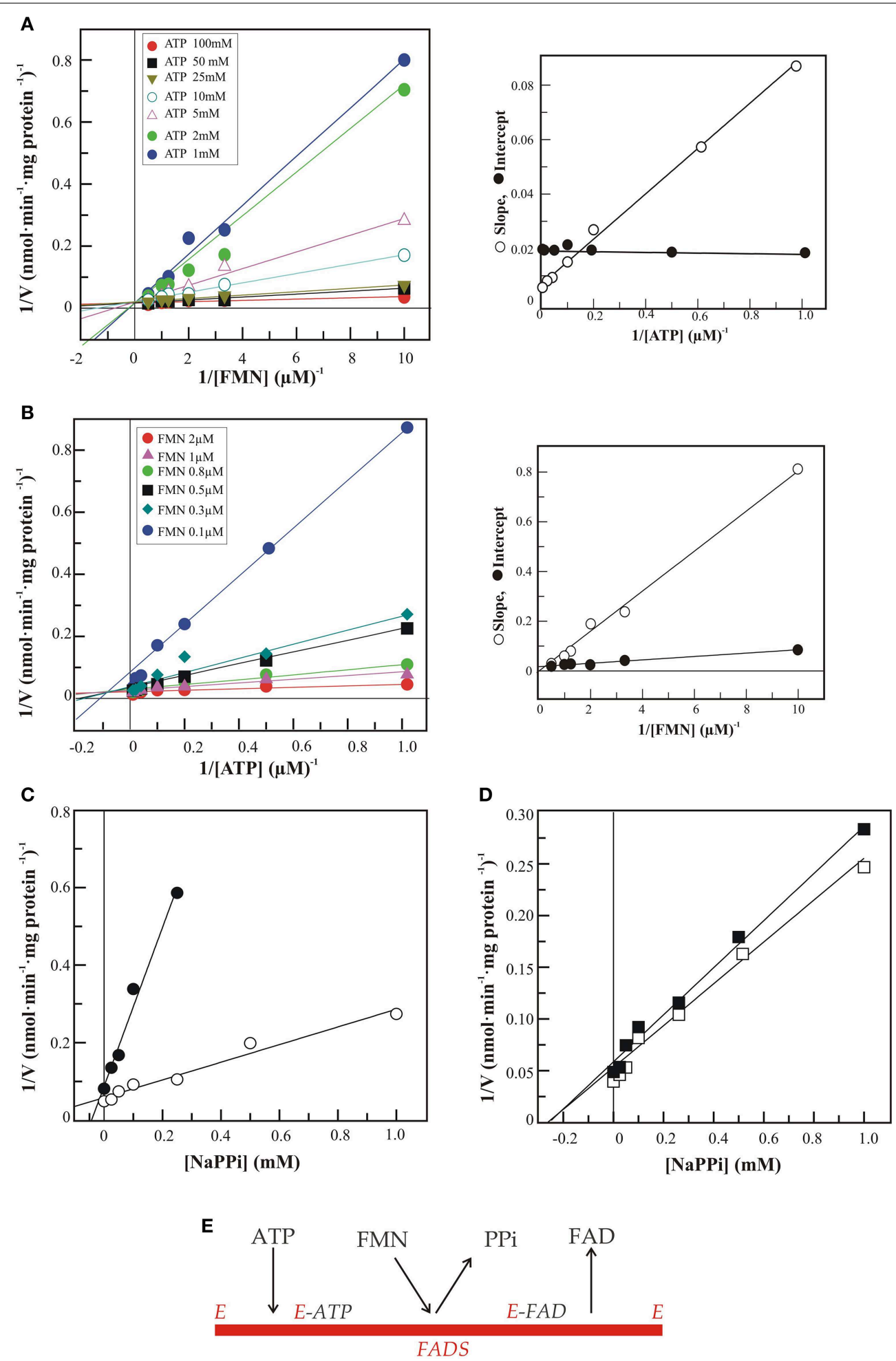

FIGURE 3 | Steady state kinetic analysis of FAD synthesis catalyzed by hFADS2. FAD synthesis rate, catalyzed by 6His-hFADS2 $(2 \mu \mathrm{g}, 35.4$ pmoli), was measured by the initial rate of fluorescence decrease $(\lambda$ ex at $450 \mathrm{~nm}, \lambda \mathrm{em}$ at $520 \mathrm{~nm}$ ) at $37^{\circ} \mathrm{C}$ in $50 \mathrm{mM}$ Tris- $\mathrm{HCl}, \mathrm{pH} 7.5$ in the presence of $5 \mathrm{mM} \mathrm{MgCl}_{2}$ and FMN and ATP at the reported concentrations. Where indicated, sodium pyrophosphate was added at the indicated concentrations. In (A) the initial rates represented by the Lineweaver-Burk plot of $1 / \nu$ vs. $1 /[\mathrm{FMN}]$ at fixed ATP concentrations. In the inset the slope and intercepts of the Linaeawever-Burk plot (with FMN as variable substrate) vs. $1 /[$ ATP]. In (B) the initial rates represented by the Lineweaver-Burk plot of $1 / \mathrm{v}$ vs. 1/[ATP] at fixed FMN concentrations. In the inset the slope and intercepts of the Linaeawever-Burk plot (with ATP as variable substrate) vs. 1/[FMN]. In (C) the Dixon plot of the inhibition by pyrophosphate at ATP $10 \mu \mathrm{M}$ and FMN $0.3 \mu \mathrm{M}(\bullet)$ or $2 \mu \mathrm{M}(\mathrm{o})$. In (D) the Dixon plot of the inhibition by pyrophosphate at FMN $2 \mu \mathrm{M}$ and ATP $10 \mu \mathrm{M}(\mathbf{\square})$ or $50 \mu \mathrm{M}(\square)$. In (E) the sequential ordered bi-bi mechanism for 6 -His-hFADS2 is represented. 
The ability of the recombinant enzyme to catalyze FAD formation and delivery to a client apo-flavoprotein was next followed by a continuous spectrophotometric assay in which newly synthesized FAD is used to reactivate apo-D-AAO as a client flavoprotein. The reconstituted activity of $\mathrm{D}-\mathrm{AAO}$ on FAD incorporation is measured as the rate of $\mathrm{NADH}$ oxidation in a coupled reaction with $\mathrm{LDH}$ (Figure 4A). Due to the time-dependence of the reconstitution reaction (Casalin et al., 1991; Barile et al., 2000), the reaction rate increases with time, reaching a maximum constant value, that was demonstrated to be proportional to the amount of the externally added cofactor (Figure 4B). In the experiment reported in Figure 4C, apo D-AAO was incubated in the absence or presence of $1 \mu \mathrm{g} 6 \mathrm{His}-\mathrm{hFADS} 2$ (which binds $1 \mathrm{~mol}$ per monomer of FADS tightly and that corresponds to $20 \mathrm{pmol}$ of the $56.5 \mathrm{kDa}$ monomer and i.e., to $20 \mathrm{pmol}$ of FAD bound to 6 His-hFADS2) as well as in the presence of equimolar amount of free FAD $(20 \mathrm{pmol})$. The rate of absorbance decrease following the addition of apo-D-AAO was equal to $0.0046 \Delta \mathrm{A} / \mathrm{min}$ in the absence of FAD addition (trace none). This rate is presumably due to a small fraction of holo-enzyme present in the assay. When $20 \mathrm{pmol}$ of FAD were added, the maximum value of the rate of absorbance decrease was equal to $0.0069 \Delta \mathrm{A} / \mathrm{min}$, in agreement with the occurrence of binding of FAD to apo-D-AAO. When adding $1 \mu \mathrm{g}$ of $6 \mathrm{His}-\mathrm{hFADS}$ to the assay, the rate of absorbance loss increased with time, reaching a rate two-fold higher than what measured with free FAD was added $(\Delta \mathrm{A} / \mathrm{min}=0.0139)$. These observations are consistent with the apo-holo transition of DAAO being accelerated by $6 \mathrm{His}-\mathrm{hFADS}$. Therefore, FADS seemed to operate not only as a synthase but also as a FAD "chaperone," that is supposed to directly interact with the client apo-flavoprotein during holoenzyme formation.

A further question was whether the expected physical interaction between FAD forming enzyme and its client could occur also with apo-flavoproteins located both in the nucleus and in the mitochondrion (Figure 5), where the enzyme could play a primary role in cellular homeostasis. The occurrence of such an interaction was previously hypothesized by different groups (Barile et al., 2013; Brizio et al., 2013). To these scope, mitochondrial dimethylglycine dehydrogenase $\left(\mathrm{Me}_{2} \mathrm{GlyDH}\right.$, EC 1.5.8.4) and nuclear lysine specific demethylase 1 (LSD1, EC 1.-.-.-) were tested as client flavoproteins. Recombinant purified hFADS was used to test the interaction with these client proteins by means of dot-blot and immuno-precipitation experiments, and to verify transfer of the cofactor to the client apoprotein, wherever possible.

\section{LSD1 as a Client Flavoprotein}

LSD1 is a nuclear FAD-containing amine oxidase, that catalyzes the demethylation of mono- and dimethylated Lys4 on histone $\mathrm{H} 3$, one of the most important recent discoveries in

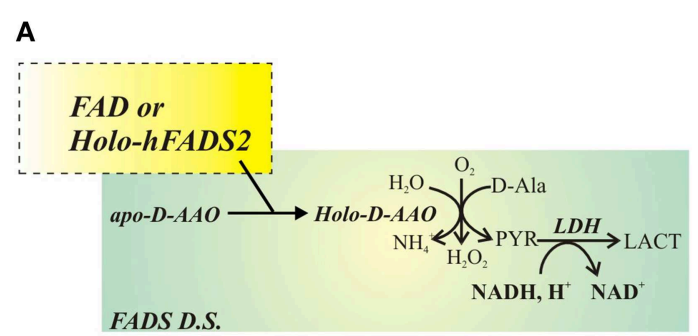

C
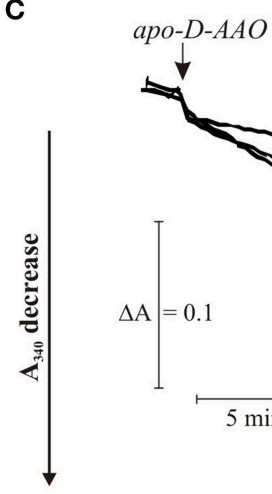

B

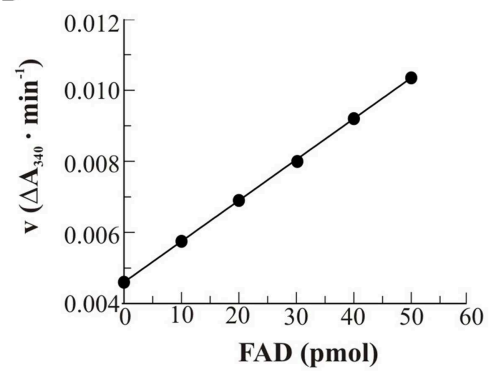

FIGURE 4 | FAD delivery from 6His-hFADS2 to the client apo-D amino acid oxidase. The release of FAD from the purified recombinant 6His-hFADS2 to apo-DAAO was assayed enzymatically, as described in Materials and Methods and schematized in (A), by measuring the activity of reconstituted holo-DAAO (derived from FAD binding to apo-DAAO). (B) Calibration curve obtained with a FAD standard. In (C) typical traces are shown. Apo-DAAO was added in the absence (none) or in the presence of purified 6His-hFADS2 (Holo-hFADS2, $1.2 \mu \mathrm{g}, 20 \mathrm{pmol})$ or in the presence of commercial FAD $(20 \mathrm{pmol})$ at $37^{\circ} \mathrm{C}$ in $100 \mu \mathrm{L}$ of $50 \mathrm{mM}$ Tris- $\mathrm{HCl}, \mathrm{pH} 7.5$. Reconstituted holo-DAAO activity was measured as described in Materials and Methods. 


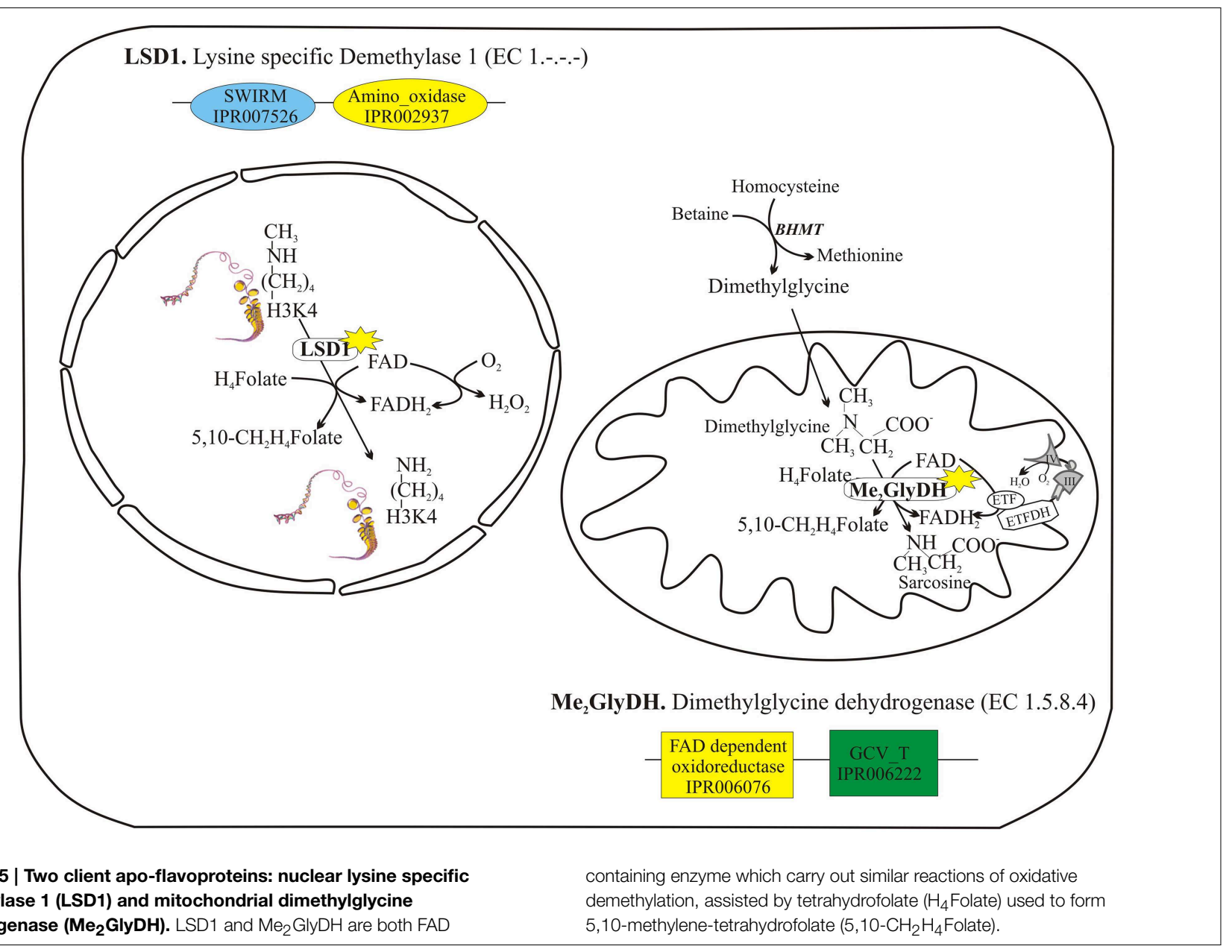

FIGURE 5 | Two client apo-flavoproteins: nuclear lysine specific demethylase 1 (LSD1) and mitochondrial dimethylglycine dehydrogenase ( $\left.\mathrm{Me}_{\mathbf{2}} \mathbf{G l y D H}\right)$. LSD1 and $\mathrm{Me}_{2} \mathrm{GlyDH}$ are both FAD demethylation, assisted by tetrahydrofolate $\left(\mathrm{H}_{4}\right.$ Folate) used to form 5,10-methylene-tetrahydrofolate $\left(5,10-\mathrm{CH}_{2} \mathrm{H}_{4}\right.$ Folate). nutri-epigenetics (Hino et al., 2012). During the course of the reaction, FAD oxidizes the lysine $\mathrm{N}$-methyl amine to lysine $\mathrm{N}$ methylimine, and $\mathrm{FADH}_{2}$ is reoxidized to FAD by molecular oxygen producing hydrogen peroxide. In the absence of tetrahydrofolate, the oxidation of methyl groups generates formaldehyde, potentially highly toxic for the nucleus, that is expected to be reduced and recycled to give S-adenosyl methionine (SAM), the main nuclear methyl donor (Tyihak et al., 1998). Recently it has been demonstrated that LSD1, as well as $\mathrm{Me}_{2} \mathrm{GlyDH}$, is able to bind tetrahydrofolate. Thus, both these reactions generate $\mathrm{N}$ 5,10-methylene-tetrahydrofolate (Figure 5) in a sort of oxidative demethylation of the substrates (Luka et al., 2011, 2014). Therefore, nuclear pool of flavins and folates seems to be strictly interconnected in controlling the crucial processes of methylation status of hystones and in nutri-epigenetics.

The LSD1 structure is well characterized. The protein consists of a classical FAD-amino oxidase domain (IPR002937) where the flavin cofactor is bound (not covalently). The folate-binding site is located in the active center in close proximity to FAD. The N-terminus contains a SWIRM domain (IPR007526), presumably responsible for nucleosome recognition (Da et al., 2006) and it is preceded by a disordered extension (Shi et al., 2004). Two isoforms are reported in Entrez Gene for humans, as products of alternative splicing of the KDM1A gene, the canonical isoform being a 852 residue long polypeptide $(92.9 \mathrm{kDa})$. A single rat isoform - corresponding to LSD1-is reported in Entrez Gene, as a 872 residue long protein $(\mathrm{Mr}=94.4 \mathrm{kDa})$ named $K d m 1 a$, exhibiting a similarity of $98 \%$ with respect to human protein. Nevertheless, a wide BLAST search in non-redundant protein sequence databases using as a query the canonical human LSD1 isoform gave an additional product of 776 amino acids $(86.1 \mathrm{kDa})$, exhibiting a $99 \%$ identity with the human protein. This isoform lacks of the first 96 aa respect to rat $K d m 1 a$ protein. A prediction made with PSORTII program, gives a score of $11 \%$ of nuclear localisation for Kdm1a and of $39 \%$ for the "similar to AOF2 protein." Kdm1a scores a $44 \%$ probability for an extracellular localisation and a $22 \%$ probability for a cytosolic one. The "similar to AOF2 protein" has a high probability to be cytosolic (52\%) and a very low probability to be localized in the mitochondrion (4\%). No cleavage sites have been identified by this bioinformatics approach.

In order to get some insight into LSD1 biogenesis, a nuclear fraction was purified from rat liver homogenate, as previously described (Giancaspero et al., 2013) and the enrichment in specific nuclear protein assessed by measuring the increase in the enzymatic activity of the nuclear enzyme nicotinamide 
mononucleotide adenylyltransferase, as described under Materials and Methods.

First of all, the existence of FADS in the nucleus was revealed by dot-blot, using a polyclonal antiserum against hFADS (antiFADS) and increasing amount of nuclear protein (50 or $100 \mu \mathrm{g})$ (Figure 6A). In parallel runs, dotted nuclear proteins were tested for the presence of LSD1 by using a commercial monoclonal antibody. The identity of the nuclear fraction was further validated by observing the enrichment of the nuclear protein LSD1 with respect to the spots obtained when 50 or $100 \mu \mathrm{g}$ of homogenate were added as a control (data not shown).

In order to investigate if the natural nuclear LSD1 protein is able to directly interact with FADS, in a parallel experiments $5 \mu \mathrm{g}$ of recombinant human 6 His-hFADS2 were dotted on the membranes, and successful dotting was tested by using the antiFADS antiserum. FADS-dotted membranes were washed and incubated for $15 \mathrm{~min}$ with nuclear proteins, and the amount of nuclear LSD1 bound to 6His-hFADS in the dots measured immunochemically in comparison with the amount of protein bound when starting from the homogenate. No 6His-hFADS2 was present in controls, and the specificity of 6His-hFADS2 /LSD1 interaction was tested by verifying the absence of actin bound to the 6His-hFADS dotted membrane (Figure 6A).

To further validate the specificity of 6His-hFADS2/ LSD1 interaction, immuno-precipitation experiments were performed and reported in Figure 6B. Following over-night incubation (according to the manufacturer-suggested procedure, see below), different anti-FADS cross-reactive bands were found in subsequent electrophoretic analysis of the products. The most evident band was found at about $38 \mathrm{kDa}$, likely being a hydrolysis product (see also Giancaspero et al., 2013). In the same fractions, after stripping procedure, an anti-LSD1 reactive band migrating at $97 \mathrm{kDa}$ was revealed (in good agreement with the molecular mass of the $\mathrm{kdm} 1 \mathrm{a}$ isoform) accompanied by a lower migrating band (apparently a hydrolysis product of about $58 \mathrm{kDa}$ ). The $97 \mathrm{kDa}$ band appeared significantly enriched in the nuclear fraction.

Purified nuclear fractions were, then, immuno-precipitated by using the anti-FADS antiserum and the immuno-precipitation kit
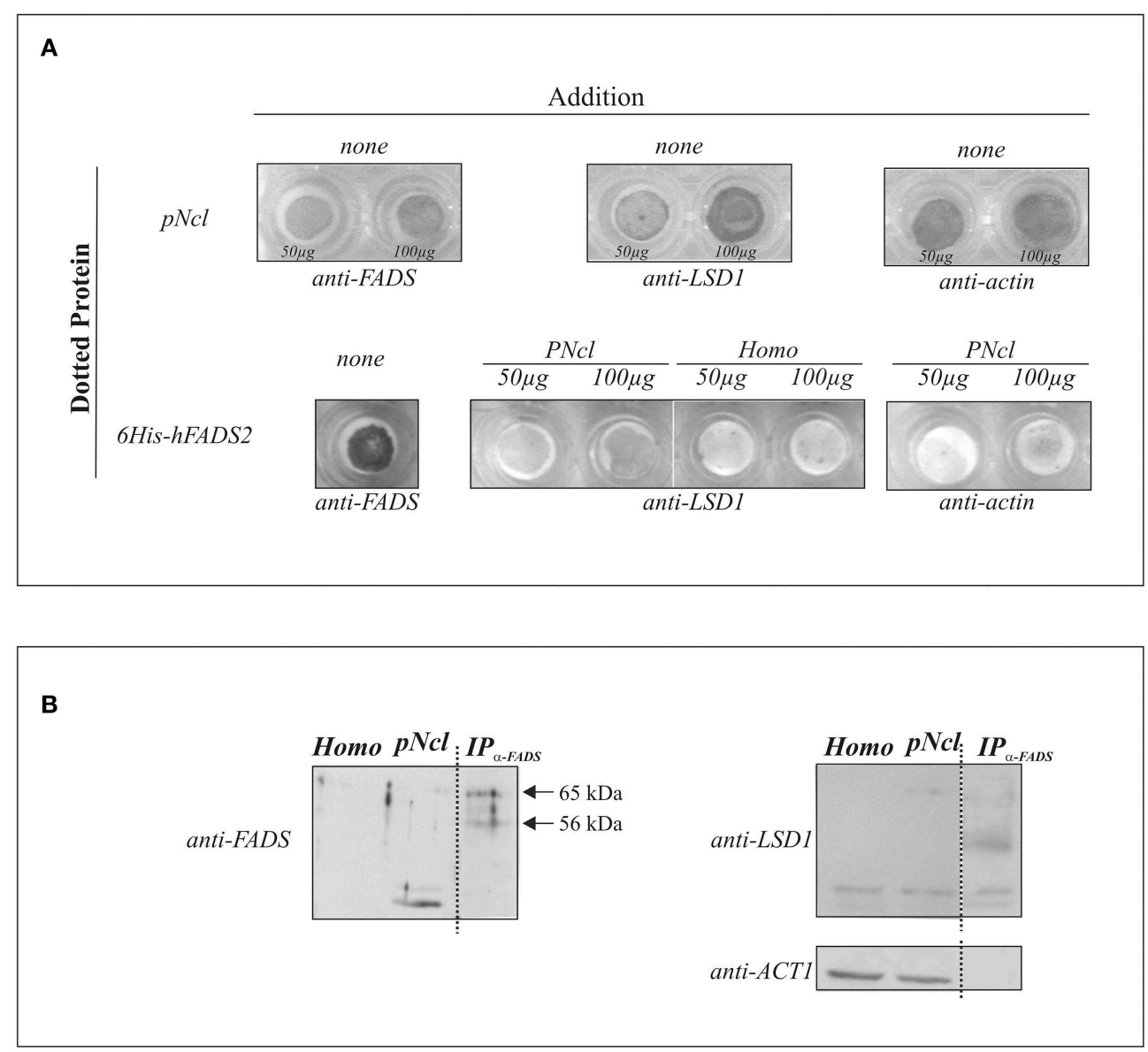

FIGURE 6 | FADS/LSD1 interaction as revealed by immunological techniques. In (A) the dot-blot assay is reported: briefly, purified rat liver nuclei (pNcl, 50 or $100 \mu \mathrm{g}$ ) resuspended in the nuclear buffer or 6His-hFADS2 $(5 \mu \mathrm{g})$ were dotted onto a nitrocellulose membrane. Where indicated $\mathrm{pNcl}(50$ or $100 \mu \mathrm{g}$ ), homogenate (homo, 50 or $100 \mu \mathrm{g}$ ) or the nuclear buffer (none) were added to the dotted membrane. Protein/protein interaction was revealed immunochmically as described in Materials and Methods. In (B) rat liver homogenate (homo, $25 \mu \mathrm{g}$ ), nuclei ( $\mathrm{pNcl}, 25 \mu \mathrm{g}$ ) and the immunoprecipitate $\left(\mathrm{IP}_{\text {anti-FADS }}\right)$ from nuclear proteins $(50 \mu \mathrm{g})$ were analyzed by immunoblotting with anti-FADS antiserum, as described in Materials and Methods. The same PVDF membrane was analyzed with the anti-LSD1 and anti-ACT1 antibodies after stripping procedure. 
Dynabeads Protein G. Immuno-precipitation resulted in a clear enrichment of the three main anti-FADS cross reacting bands located in the $65-56 \mathrm{kDa}$ range, as expected from the predicted molecular mass of rat FADSs (Giancaspero et al., 2013). The same fraction was also revealed by using the anti-LSD1 antibody. Different cross reactive bands were immune-precipitated together with FADS, and one of these bands migrates at about $80 \mathrm{kDa}$. ESIMS/MS analysis will be performed to identify whether the $80 \mathrm{kDa}$ band is a hydrolytic product of $\mathrm{kdm} l$ a or rather the protein similar to AOF2. Thus, the emerging picture favors the proposal of a physical interaction between the nuclear apo-flavoprotein LSD1 and FADS, an interaction of high relevance for attachment of a FAD cofactor during enzyme biogenesis.

\section{$\mathrm{Me}_{2} \mathrm{GlyDH}$ as a Client Flavoprotein}

The mechanism of flavoprotein biogenesis is of particular relevance in mitochondria, since they are the main site of localisation of flavoenzymes bearing both covalently-bound FAD and noncovalently bound FAD or FMN (Heikal, 2010; Barile et al., 2013;
Lienhart et al., 2013). As far as the mitochondrial flavoproteome is concerned we focused our attention on $\mathrm{Me}_{2} \mathrm{GlyDH}$, a key enzyme of folate one-carbon metabolism and choline catabolism (Figure 5), located in the mitochondrial matrix. In the presence of tetrahydrofolate (THF), $\mathrm{Me}_{2} \mathrm{GlyDH}$ catalyzes the oxidative demethylation of dimethylglycine to yield sarcosine and 5,10methylene-THF. In the natural mature $\mathrm{Me}_{2} \mathrm{GlyDH}$ the FAD cofactor is covalently linked to the enzyme via a histidyl(N3)-( $8 \alpha)$ FAD linkage occurring at His84 (Cook et al., 1985). $\mathrm{Me}_{2} \mathrm{GlyDH}$ is synthesized in the cytosol as a precursor protein containing an $\mathrm{N}$-terminal extra-sequence, which is removed in the organelle by the mitochondrial processing peptidase (MPP) (Otto et al., 1996). The flavin attachment event in vivo occurs in mitochondria before removal of the pre-sequence (Brizio et al., 2002).

Both the mature $\left(\mathrm{mMe}_{2} \mathrm{GlyDH}\right)$ and the precursor ( $\mathrm{pMe}_{2} \mathrm{GlyDH}$ ) forms of rat $\mathrm{Me}_{2} \mathrm{GlyDH}$ were produced in Escherichia coli as, respectively, a N-terminally and C-terminally 6-His-tagged fusion protein (Brizio et al., 2004, 2008). Flavinylation of the in vitro synthesized apo- $\mathrm{Me}_{2} \mathrm{GlyDH}$ protein seemed

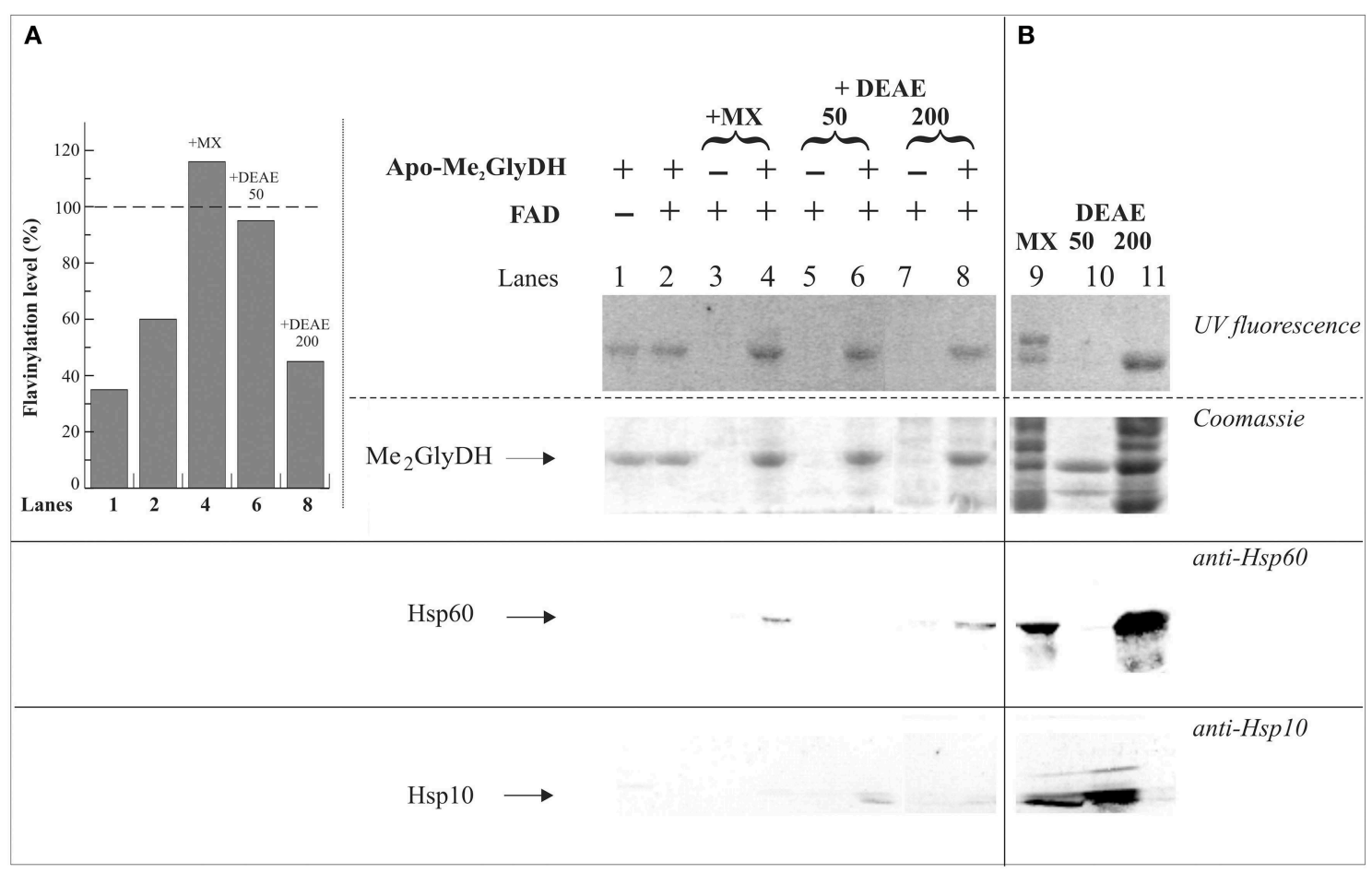

FIGURE 7 | Evidences of $\mathrm{Me}_{2} \mathrm{GlyDH}$ flavinylation and identification of possible interactors. In (A) $6 \mathrm{His}-\mathrm{apo}-\mathrm{Me}_{2} \mathrm{GlyDH}$ $(10 \mu \mathrm{g})$ was incubated at $37^{\circ} \mathrm{C}$ in flavinylation medium made of $50 \mathrm{mM}$ Tris- $\mathrm{HCl}, \mathrm{pH} 7.5,5 \mathrm{mM} \mathrm{MgCl}_{2} 5 \mathrm{mM}, 0.5 \%$ Triton X-100, $5 \mathrm{mM}$ ATP, in the absence or presence of $20 \mu \mathrm{M}$ FAD. Where indicated rat liver mitochondrial matrix (MX) (lane 4) or $M X$ fractions eluted onto a DEAE-Sephacel column with 50 and $200 \mathrm{mM} \mathrm{NaCl}$ (D50 and D200 fractions, $100 \mu \mathrm{g}$ each, lanes 6 and 8, respectively) were added to the reaction mixture. As a control the same matrix and DEAE fractions were incubated in the same experimental condition in the absence of apo- $\mathrm{Me}_{2} \mathrm{GlyDH}$ and in the presence of $20 \mu \mathrm{M}$ FAD (lanes 3, 5, 7). After $1 \mathrm{~h}$ incubation, each sample was passed on a Ni-Chelating Sepharose to re-isolate the recombinant
6 His-apo- $\mathrm{Me}_{2} \mathrm{GlyDH}$ and possible interactors. After washing with $50 \mathrm{mM}$ imidazole, bound $6 \mathrm{His}-\mathrm{apo}-\mathrm{Me}_{2} \mathrm{GlyDH}$ and its possible interactors were eluted with $500 \mathrm{mM}$ imidazole, precipitated with acetone, and analyzed by SDS-PAGE. The flavin fluorescence of SDS-PAGE separated proteins was visualized by UV irradiation of the unstained gel soaked in $10 \%$ acetic acid. Protein bands were then stained with Coomassie Brilliant Blue. The interactors were searched for by immunoblotting analysis carried out using anti Hsp6O and Hsp10 antibodies. The flavinylation level of $\mathrm{Me}_{2} \mathrm{GlyDH}$ (inset) was estimated through image analysis as described in Materials and Methods. In (B) matrix and fractions D50 and D200 (100 $\mu \mathrm{g}$ each, lanes 9-11) from ion-exchange chromatography were analyzed by SDS-PAGE and immunoblotting as described in (A). 

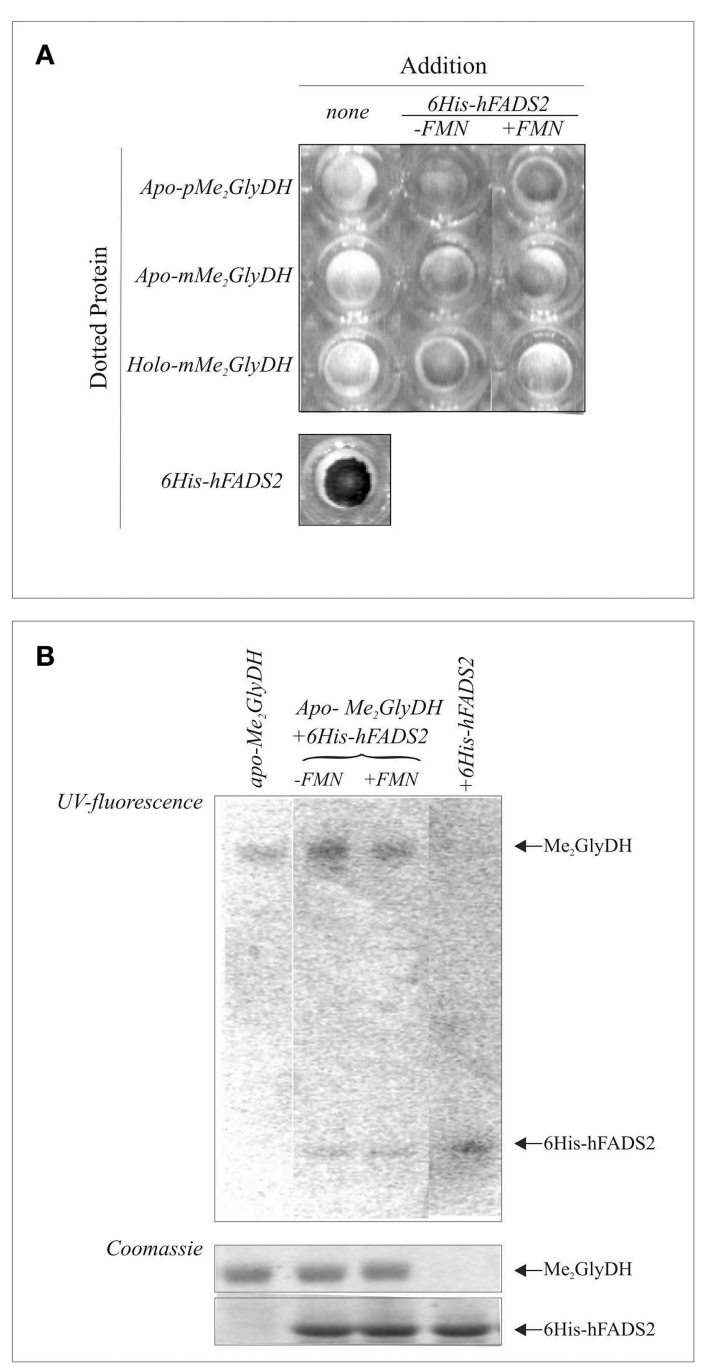

FIGURE 8 | Evidences of physical interaction and cofactor release from hFADS to the client $\mathbf{M e}_{\mathbf{2}}$ GlyDH. In (A) purified either precursor (p) or mature (m) form of apo- and holo-Me ${ }_{2} \mathrm{GlyDHs}$ (1 $\mu \mathrm{g}$ each) were dotted onto a nitrocellulose membrane and incubated with purified recombinant human 6 His-FADS2 in the presence of $5 \mathrm{mM}$ ATP and $5 \mathrm{mM} \mathrm{MgCl}_{2}$. Where indicated $20 \mu \mathrm{M}$ FMN was added. After $30 \mathrm{~min}$ incubation at $37^{\circ} \mathrm{C}$ the membrane was washed and probed with an anti-hFADS antiserum. In (B) purified recombinant apo-pMe ${ }_{2} \mathrm{GlyDH}(1 \mu \mathrm{g})$ was incubated at $37^{\circ} \mathrm{C}$ in the presence or absence of recombinant 6 His-hFADS2 $(3.3 \mu \mathrm{g})$ in $40 \mathrm{mM}$ Hepes buffer $\mathrm{pH} 7.4$ containing $5 \mathrm{mM}$ ATP and $5 \mathrm{mM} \mathrm{MgCl}_{2}$. FMN $(20 \mu \mathrm{M})$ was added where indicated. As a control, 6His-hFADS2 $(3.3 \mu \mathrm{g})$ was incubated in the same conditions, but in

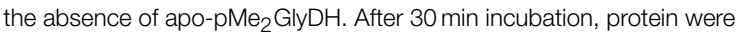
denatured with the addition of sample buffer, boiled at $95^{\circ} \mathrm{C}$ and analyzed by SDS-PAGE. The flavin fluorescence of proteins was visualized by UV irradiation of the unstained gel soaked in 10\% acetic acid. Proteins were then stained with Coomassie Brilliant Blue.

to proceed spontaneously in the presence of FAD, in line with an autocatalytic process (Otto et al., 1996). Nevertheless, the rate of $\mathrm{Me}_{2} \mathrm{GlyDH}$ holoenzyme formation was found to be stimulated by protein factor(s) localized in mitochondrial matrix, that were tentatively named mitochondrial flavinylation stimulating factor(s) (mtFSF) (Brizio et al., 2000, 2002). mtFSF(s) elutes at $50 \mathrm{mM} \mathrm{NaCl}$ (DEAE50) following fractionation of the mitochondrial matrix by ionic-exchange chromatography on a DEAE-Sephacel column (Brizio et al., 2000, 2013).

A simple, rapid and direct method for determining the flavinylation level of the $\mathrm{Me}_{2} \mathrm{GlyDH}$ consists in revealing holoenzyme flavin fluorescence upon irradiation with UV light of SDS-PAGE gels provides (Brizio et al., 2004, 2008). Rat liver mitochondrial matrix gave two fluorescent native bands corresponding to the two covalently flavinylated mitochondrial enzymes sarcosine dehydrogenase and $\mathrm{Me}_{2} \mathrm{GlyDH}$ (Figure 7B, lane 9). The native $\mathrm{Me}_{2} \mathrm{GlyDH}$ was eluted as a fluorescent band at $200 \mathrm{mM} \mathrm{NaCl}$ (DEAE200) following DEAE-Sephacel chromatography of mitochondrial matrix. Immunoblotting analysis of DEAE fractions carried out with anti-Hsp60 antibodies revealed that Hsp60 was mainly recovered in the DEAE200 fraction and was completely absent in materials eluted at lower ionic strength $(50 \mathrm{mM} \mathrm{NaCl}$, DEAE50). This confirms that mtFSF differs from the matrix chaperone Hsp60 (Brizio et al., 2000). Thus, a matrix component other than Hsp60 might assist flavinylation of apo- $\mathrm{Me}_{2} \mathrm{GlyDH}$. Instead, we gathered immunoblotting evidence of the Hsp10 cochaperone being present in the DEAE50 fraction together with the naturally occurring FADS (not shown).

In Figure 7A, to search for possible Me2GlyDH interactors during flavinylation reaction, "re-binding" experiments were performed Briefly, His- $\mathrm{Me}_{2} \mathrm{GlyDH}$ was re-isolated on Nichelating Sepharose after a flavinylation step (Brizio et al., 2013). UV fluorescence measurements (compare lanes 1-4, and histogram in the inset) clearly confirm that mitochondrial matrix is able to stimulate apo- $\mathrm{Me}_{2} \mathrm{GlyDH}$ flavinylation (Brizio et al., 2013). This stimulation is observed with the DEAE50 fraction, but not with the DEAE200 one. Both the chaperone Hsp60 and the co-chaperone Hsp10 interact with 6-His- $\mathrm{Me}_{2} \mathrm{GlyDH}$, as revealed by immunoblotting analysis (Figure 7), therefore they both are expected to participate in the flavinylation machinery. Therefore, the finding that the matrix flavinylation stimulating activity elutes in the same chromatographic fraction that contains mtFADS (Brizio et al., 2013), is prompting us to propose that-besides synthesizing the cofactor-FADS provide a chaperoning activity during $\mathrm{Me}_{2} \mathrm{GlyDH}$ biogenesis.

To further demonstrate that recombinant hFADS physically interacts with the $\mathrm{Me}_{2} \mathrm{GlyDH}$ client, protein-protein interaction was analyzed by dot-blot experiments in which the purified recombinant $\mathrm{Me}_{2} \mathrm{GlyDH}(1 \mu \mathrm{g})$ was dotted onto the membrane and incubated with purified recombinant human 6His-hFADS2 in the presence of ATP $(5 \mathrm{mM})$ and, when indicated, of FMN $(20 \mu \mathrm{M})$. After $30 \mathrm{~min}$ incubation at $37^{\circ} \mathrm{C}$ the membrane was washed and probed with an anti-hFADS antibody. These experiments, reported in Figure 8A, indicated that FADS interacts with both the precursor $\left(\mathrm{pMe}_{2} \mathrm{GlyDH}\right)$ and mature $\left(\mathrm{mMe}_{2} \mathrm{GlyDH}\right)$ form of the acceptor protein.

To test whether direct transfer of FAD to the client protein, purified recombinant apo-pMe $\mathrm{GlyDH}_{2}$ was incubated in the presence of ATP with purified recombinant hFADS, that binds tightly $1 \mathrm{~mol} \mathrm{FAD/mol} \mathrm{protein.} \mathrm{After} 30 \mathrm{~min}$ incubation, proteins were analyzed by SDS-PAGE, to follow holoenzyme formation. The unstained gel was analyzed to detect flavin fluorescence 


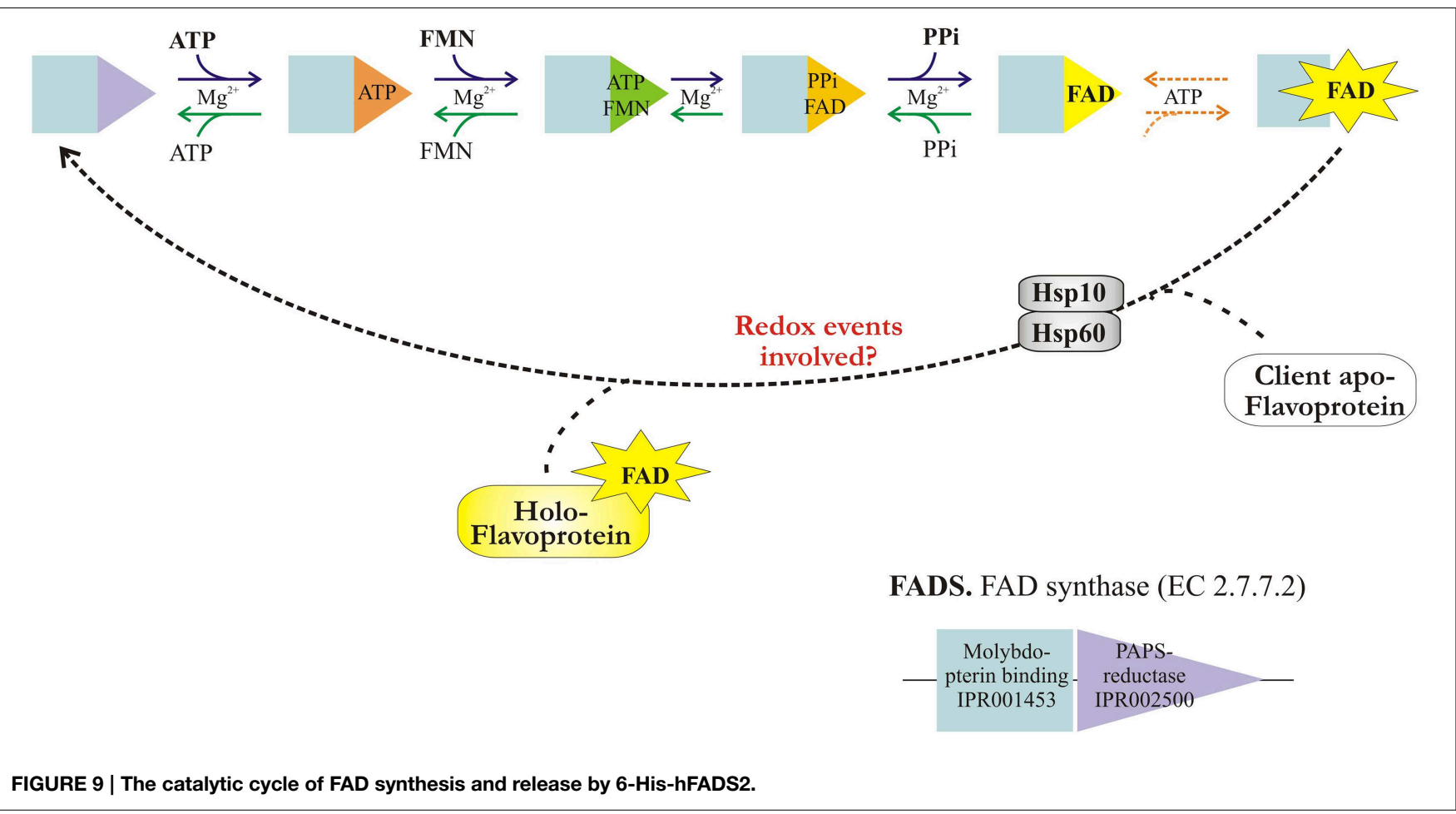

prior to protein staining. As shown in Figure 8B, hFADS loses its fluorescence upon incubation with apo- $\mathrm{Me}_{2} \mathrm{GlyDH}$ while simultaneously promoting the flavinylation of apo- $\mathrm{Me}_{2} \mathrm{GlyDH}$. The yield in flavinylated $\mathrm{Me}_{2} \mathrm{GlyDH}$ increased when FMN was added together with ATP.

Based on these and our previous results, we propose that mitochondrial FADS, besides synthesizing FAD, acts in mitochondria as a "FAD chaperone" recognizing nascent apo-flavoproteins and promoting their flavinylation.

\section{Conclusion}

Experiments described here confirmed the nuclear localization of FADS in human cells and demonstrate a direct interaction between the FAD forming machinery and the nuclear client flavoprotein LSD1, thus shading light on the possible role of flavin cofactor homeostasis in epigenetic control.

Using the mitochondrial apo-flavoenzyme $\mathrm{Me}_{2} \mathrm{GlyDH}$, we observed a direct protein-protein interaction and the cofactor transfer from the donor to the apo-protein acceptor, occurring in

\section{References}

Bacher, A., Eberhardt, S., Fischer, M., Kis, K., and Richter, G. (2000). Biosynthesis of vitamin b2 (riboflavin). Annu. Rev. Nutr. 20, 153-167. doi: 10.1146/annurev.nutr.20.1.153

Bafunno, V., Giancaspero, T. A., Brizio, C., Bufano, D., Passarella, S., Boles, E., et al. (2004). Riboflavin uptake and FAD synthesis in Saccharomyces cerevisiae mitochondria: involvement of the Flx1p carrier in FAD export. J. Biol. Chem. 279, 95-102. doi: 10.1074/jbc.M308230200 a sort of "FAD-chaperoning" action played by hFADS per se. The role of Hsp60/Hsp10 and a possible control exerted by the redox status of the cofactor during its transfer from FADS to substrate proteins is an interesting matter of future investigation.

The ordered bi-bi mechanism of FAD synthesis together with the proposal of a flavinylation pathway involving hFADS are presented in Figure 9.

\section{Acknowledgments}

The authors are grateful to Prof. C. Indiveri (Università della Calabria) for the interpretation and critical reading of the steady state kinetics data. The assistance of Dr. A. Miccolis and Dr. V. C. Liuzzi in the early stages of this work, and the technical assistance of V. Giannoccaro (Università degli Studi di Bari “Aldo Moro") is also gratefully acknowledged. This work was supported by PONricerca e competitività 2007-2013 (project 01_00937: “Modelli sperimentali biotecnologici integrati per la produzione ed il monitoraggio di biomolecole di interesse per la salute dell'uomo," to M.B). 
Barile, M., Giancaspero, T. A., Brizio, C., Panebianco, C., Indiveri, C., Galluccio, M., et al. (2013). Biosynthesis of flavin cofactors in man: implications in health and disease. Curr. Pharm. Des. 19, 2649-2675. doi: $10.2174 / 1381612811319140014$

Barile, M., Passarella, S., Bertoldi, A., and Quagliariello, E. (1993). Flavin adenine dinucleotide synthesis in isolated rat liver mitochondria caused by imported flavin mononucleotide. Arch. Biochem. Biophys. 305, 442-447. doi: 10.1006/abbi.1993.1444

Bonomi, F., Iametti, S., Morleo, A., Ta, D., and Vickery, L. E. (2008). Studies on the mechanism of catalysis of iron-sulfur cluster transfer from IscU[2Fe2S] by HscA/HscB chaperones. Biochemistry 47, 12795-12801. doi: 10.1021/bi801565j

Bradford, M. M. (1976). A rapid and sensitive method for the quantitation of microgram quantities of protein utilizing the principle of protein-dye binding. Anal. Biochem. 72, 248-254. doi: 10.1016/0003-2697(76)90527-3

Brizio, C., Barile, M., and Brandsch, R. (2002). Flavinylation of the precursor of mitochondrial dimethylglycine dehydrogenase by intact and solubilised mitochondria. FEBS Lett. 522, 141-146. doi: 10.1016/S0014-5793(02)02927-7

Brizio, C., Brandsch, R., Bufano, D., Pochini, L., Indiveri, C., and Barile, M. (2004). Over-expression in Escherichia coli, functional characterization and refolding of rat dimethylglycine dehydrogenase. Protein Expr. Purif. 37, 434-442. doi: 10.1016/j.pep.2004.06.011

Brizio, C., Brandsch, R., Douka, M., Wait, R., and Barile, M. (2008). The purified recombinant precursor of rat mitochondrial dimethylglycine dehydrogenase binds FAD via an autocatalytic reaction. Int. J. Biol. Macromol. 42, 455-462. doi: 10.1016/j.ijbiomac.2008.03.001

Brizio, C., Galluccio, M., Wait, R., Torchetti, E. M., Bafunno, V., Accardi, R., et al. (2006). Over-expression in Escherichia coli and characterization of two recombinant isoforms of human FAD synthetase. Biochem. Biophys. Res. Commun. 344, 1008-1016. doi: 10.1016/j.bbrc.2006.04.003

Brizio, C., Giancaspero, T. A., Brandsch, R., and Barile, M. (2013). "Mitochondriaassisted flavinylation of recombinant rat dimethylglycine dehydrogenase," Proceedings of 17th International Symposium on Flavins and Flavoproteins, IUMBM S3/2011, 24-29 July 2011, eds S. Miller, R. Hille, and B. Palfey (Berkeley, CA), 493-501. Publisher Lulu.com, NC.

Brizio, C., Otto, A., Brandsch, R., Passarella, S., and Barile, M. (2000). A protein factor of rat liver mitochondrial matrix involved in flavinylation of dimethylglycine dehydrogenase. Eur. J. Biochem. 267, 4346-4354. doi: 10.1046/j.14321327.2000.01464.x

Bruni, F., Manzari, C., Filice, M., Loguercio Polosa, P., Colella, M., Carmone, C., et al. (2012). D-MTERF5 is a novel factor modulating transcription in Drosophila mitochondria. Mitochondrion 12, 492-499. doi: 10.1016/j.mito.2012.06.010

Cardone, A., Lopez, F., Affortunato, F., Busco, G., Hofer, A. M., Mallamaci, R., et al. (2012). An aryleneethynylene fluorophore for cell membrane staining. Biochim. Biophys. Acta 1818, 2808-2817. doi: 10.1016/j.bbamem.2012.06.011

Casalin, P., Pollegioni, L., Curti, B., and Pilone Simonetta, M. (1991). A study on apoenzyme from Rhodotorula gracilis D-amino acid oxidase. Eur. J. Biochem. 197, 513-517. doi: 10.1111/j.1432-1033.1991.tb15939.x

Clealand, W. W. (1970). The Enzymes, 3rd Edn. Academic Press, 1-64.

Cook, R. J., Misono, K. S., and Wagner, C. (1985). The amino acid sequences of the flavin-peptides of dimethylglycine dehydrogenase and sarcosine dehydrogenase from rat liver mitochondria. J. Biol. Chem. 260, 12998-13002.

Da, G., Lenkart, J., Zhao, K., Shiekhattar, R., Cairns, B. R., and Marmorstein, R. (2006). Structure and function of the SWIRM domain, a conserved protein module found in chromatin regulatory complexes. Proc. Natl. Acad. Sci. U.S.A. 103, 2057-2062. doi: 10.1073/pnas.0510949103

Foley, A. R., Menezes, M. P., Pandraud, A., Gonzalez, M. A., Al-Odaib, A., Abrams, A. J., et al. (2014). Treatable childhood neuronopathy caused by mutations in riboflavin transporter RFVT2. Brain 137, 44-56. doi: 10.1093/brain/ awt315

Galluccio, M., Brizio, C., Torchetti, E. M., Ferranti, P., Gianazza, E., Indiveri, C., et al. (2007). Over-expression in Escherichia coli, purification and characterization of isoform 2 of human FAD synthetase. Protein Expr. Purif. 52, 175-181. doi: 10.1016/j.pep.2006.09.002

Gerbino, A., Maiellaro, I., Carmone, C., Caroppo, R., Debellis, L., Barile, M., et al. (2012). Glucose increases extracellular [Ca2+] in rat insulinoma (INS-1E) pseudoislets as measured with Ca2+-sensitive microelectrodes. Cell Calcium 51, 393-401. doi: 10.1016/j.ceca.2012.01.002
Giancaspero, T. A., Busco, G., Panebianco, C., Carmone, C., Miccolis, A., Liuzzi, G. M., et al. (2013). FAD synthesis and degradation in the nucleus create a local flavin cofactor pool. J. Biol. Chem. 288, 29069-29080. doi: 10.1074/jbc.M113.500066

Giancaspero, T. A., Locato, V., de Pinto, M. C., De Gara, L., and Barile, M. (2009). The occurrence of riboflavin kinase and FAD synthetase ensures FAD synthesis in tobacco mitochondria and maintenance of cellular redox status. FEBS J. 276, 219-231. doi: 10.1111/j.1742-4658.2008.06775.x

Haack, T. B., Makowski, C., Yao, Y., Graf, E., Hempel, M., Wieland, T., et al. (2012). Impaired riboflavin transport due to missense mutations in SLC52A2 causes Brown-Vialetto-Van Laere syndrome. J. Inherit. Metab. Dis. 35, 943-948. doi: $10.1007 /$ s10545-012-9513-y

Heikal, A. A. (2010). Intracellular coenzymes as natural biomarkers for metabolic activities and mitochondrial anomalies. Biomark. Med. 4, 241-263. doi: 10.2217/bmm.10.1

Hino, S., Sakamoto, A., Nagaoka, K., Anan, K., Wang, Y., Mimasu, S., et al. (2012). FAD-dependent lysine-specific demethylase-1 regulates cellular energy expenditure. Nat. Commun. 3:758. doi: 10.1038/ncomms1755

Huerta, C., Borek, D., Machius, M., Grishin, N. V., and Zhang, H. (2009). Structure and mechanism of a eukaryotic FMN adenylyltransferase. J. Mol. Biol. 389, 388-400. doi: 10.1016/j.jmb.2009.04.022

Joosten, V., and van Berkel, W. J. (2007). Flavoenzymes. Curr. Opin. Chem. Biol. 11, 195-202. doi: 10.1016/j.cbpa.2007.01.010

Kim, H. J., and Winge, D. R. (2013). Emerging concepts in the flavinylation of succinate dehydrogenase. Biochim. Biophys. Acta 1827, 627-636. doi: 10.1016/j.bbabio.2013.01.012

Lienhart, W. D., Gudipati, V., and Macheroux, P. (2013). The human flavoproteome. Arch. Biochem. Biophys. 535, 150-162. doi: 10.1016/j.abb.2013.02.015

Lin, J., Diamanduros, A., Chowdhury, S. A., Scelsa, S., Latov, N., and Sadiq, S. A. (2009). Specific electron transport chain abnormalities in amyotrophic lateral sclerosis. J. Neurol. 256, 774-782. doi: 10.1007/s00415-009-5015-8

Liuzzi, V. C., Giancaspero, T. A., Gianazza, E., Banfi, C., Barile, M., and De Giorgi, C. (2012). Silencing of FAD synthase gene in Caenorhabditis elegans upsets protein homeostasis and impacts on complex behavioral patterns. Biochim. Biophys. Acta 1820, 521-531. doi: 10.1016/j.bbagen.2012.01.012

Luka, Z., Moss, F., Loukachevitch, L. V., Bornhop, D. J., and Wagner, C. (2011). Histone demethylase LSD1 is a folate-binding protein. Biochemistry 50, 4750-4756. doi: 10.1021/bi200247b

Luka, Z., Pakhomova, S., Loukachevitch, L. V., Calcutt, M. W., Newcomer, M. E., and Wagner, C. (2014). Crystal structure of the histone lysine specific demethylase LSD1 complexed with tetrahydrofolate. Protein Sci. 23, 993-998. doi: 10.1002 /pro.2469

Miccolis, A., Galluccio, M., Nitride, C., Giancaspero, T. A., Ferranti, P., Iametti, S., et al. (2014). Significance of redox-active cysteines in human FAD synthase isoform 2. Biochim. Biophys. Acta 1844, 2086-2095. doi: 10.1016/j.bbapap.2014.08.005

Nabokina, S. M., Subramanian, V. S., and Said, H. M. (2012). Effect of clinical mutations on functionality of the human riboflavin transporter-2 (hRFT-2). Mol. Genet. Metab. 105, 652-657. doi: 10.1016/j.ymgme.2011.12.021

Oka, M., and McCormick, D. B. (1987). Complete purification and general characterization of FAD synthetase from rat liver. J. Biol. Chem. 262, 7418-7422.

Orsomando, G., Cialabrini, L., Amici, A., Mazzola, F., Ruggieri, S., Conforti, L., et al. (2012). Simultaneous single-sample determination of NMNAT isozyme activities in mouse tissues. PLoS ONE 7:e53271. doi: 10.1371/journal.pone.0053271

Otto, A., Stoltz, M., Sailer, H. P., and Brandsch, R. (1996). Biogenesis of the covalently flavinylated mitochondrial enzyme dimethylglycine dehydrogenase. J. Biol. Chem. 271, 9823-9829. doi: 10.1074/jbc.271.16.9823

Padovani, D., and Banerjee, R. (2009). A rotary mechanism for coenzyme $\mathrm{B}(12)$ synthesis by adenosyltransferase. Biochemistry 48, 5350-5357. doi: 10.1021/bi900454s

Pallotta, M. L., Brizio, C., Fratianni, A., De Virgilio, C., Barile, M., and Passarella, S. (1998). Saccharomyces cerevisiae mitochondria can synthesise FMN and FAD from externally added riboflavin and export them to the extramitochondrial phase. FEBS Lett. 428, 245-249. doi: 10.1016/S0014-5793(98) 00544-4

Patel, M., Vadlapatla, R. K., Pal, D., and Mitra, A. K. (2012). Molecular and functional characterization of riboflavin specific transport system 
in rat brain capillary endothelial cells. Brain Res. 1468, 1-10. doi: 10.1016/j.brainres.2012.05.052

Schindelin, J., Arganda-Carreras, I., Frise, E., Kaynig, V., Longair, M., Pietzsch, T., et al. (2012). Fiji: an open-source platform for biological-image analysis. Nat. Methods 9, 676-682. doi: 10.1038/nmeth.2019

Shi, Y., Lan, F., Matson, C., Mulligan, P., Whetstine, J. R., Cole, P. A., et al. (2004). Histone demethylation mediated by the nuclear amine oxidase homolog LSD1. Cell 119, 941-953. doi: 10.1016/j.cell.2004.12.012

Srour, M., Putorti, M. L., Schwartzentruber, J., Bolduc, V., Shevell, M. I., Poulin, C., et al. (2014). Mutations in riboflavin transporter present with severe sensory loss and deafness in childhood. Muscle Nerve 50, 775-779. doi: $10.1002 /$ mus. 24224

Stojanovski, D., Muller, J. M., Milenkovic, D., Guiard, B., Pfanner, N., and Chacinska, A. (2008). The MIA system for protein import into the mitochondrial intermembrane space. Biochim. Biophys. Acta 1783, 610-617. doi: 10.1016/j.bbamcr.2007.10.004

Thorpe, C., Hoober, K. L., Raje, S., Glynn, N. M., Burnside, J., Turi, G. K., et al. (2002). Sulfhydryl oxidases: emerging catalysts of protein disulfide bond formation in eukaryotes. Arch. Biochem. Biophys. 405, 1-12. doi: 10.1016/S00039861(02)00337-5

Tonazzi, A., Mantovani, C., Colella, M., Terenghi, G., and Indiveri, C. (2013). Localization of mitochondrial carnitine/acylcarnitine translocase in sensory neurons from rat dorsal root ganglia. Neurochem. Res. 38, 2535-2541. doi: 10.1007/s11064-013-1168-Z

Torchetti, E. M., Bonomi, F., Galluccio, M., Gianazza, E., Giancaspero, T. A., Iametti, S., et al. (2011). Human FAD synthase (isoform 2): a component of the machinery that delivers FAD to apo-flavoproteins. FEBS J. 278, 4434-4449. doi: $10.1111 /$ j.1742-4658.2011.08368.x
Torchetti, E. M., Brizio, C., Colella, M., Galluccio, M., Giancaspero, T. A., Indiveri, C., et al. (2010). Mitochondrial localization of human FAD synthetase isoform 1. Mitochondrion 10, 263-273. doi: 10.1016/j.mito.2009.12.149

Tu, B. P., Ho-Schleyer, S. C., Travers, K. J., and Weissman, J. S. (2000). Biochemical basis of oxidative protein folding in the endoplasmic reticulum. Science 290, 1571-1574. doi: 10.1126/science.290.5496.1571

Tyihak, E., Albert, L., Nemeth, Z. I., Katay, G., Kiraly-Veghely, Z., and Szende, B. (1998). Formaldehyde cycle and the natural formaldehyde generators and capturers. Acta Biol. Hung. 49, 225-238.

Yao, Y., Yonezawa, A., Yoshimatsu, H., Masuda, S., Katsura, T., and Inui, K. (2010). Identification and comparative functional characterization of a new human riboflavin transporter hRFT3 expressed in the brain. J. Nutr. 140, 1220-1226. doi: $10.3945 /$ jn.110.122911

Ye, H., and Rouault, T. A. (2010). Human iron-sulfur cluster assembly, cellular iron homeostasis, and disease. Biochemistry 49, 4945-4956. doi: 10.1021/ bi1004798

Conflict of Interest Statement: The authors declare that the research was conducted in the absence of any commercial or financial relationships that could be construed as a potential conflict of interest.

Copyright (c) 2015 Giancaspero, Colella, Brizio, Difonzo, Fiorino, Leone, Brandsch, Bonomi, Iametti and Barile. This is an open-access article distributed under the terms of the Creative Commons Attribution License (CC BY). The use, distribution or reproduction in other forums is permitted, provided the original author (s) or licensor are credited and that the original publication in this journal is cited, in accordance with accepted academic practice. No use, distribution or reproduction is permitted which does not comply with these terms. 